\title{
MAN.I2 - Technology foresight for the identification of new targets, opportunities and partnerships in plant-based platforms
}

\author{
Diana Praia Borges Freire ${ }^{1 *}$; Lívia Rubatino de Faria ${ }^{1}$; Tamiris Azamor da Costa Barros; \\ Milena Mouta Verdan França Carvalho'; Christiane de Fatima Silva Marques ${ }^{1}$; Marilza Batista \\ Correa $^{1}$; Beatriz de Castro Fialho ${ }^{1}$.
}

1Fiocruz/Bio-Manguinhos.

Introduction: As an example of many of technology foresight (TF) applications, it can help an organization to identify areas to concentrate research and development investments in short, medium and long terms. Since 2012, Bio-Manguinhos (BM) has been investing in plant-based technologies. This is an emerging field, with few registered products and only one in the market. Therefore, establishing a systematic TF will contribute to BM' decisionmaking for target selection and project prioritization to be further advanced at the Plant Based Technology Center.

Objective: To present the results of ongoing in-house TF initiatives in molecular pharming regarding the design of the process, as well the data and analyses so far obtained about the current scenario and trends in the area.

Methodology: To establish and design a TF process, we identified the main areas and business processes and interviewed main stakeholders. Regarding the application of TF methods, we used Medtrack ${ }^{\circledast}$ to identify the pipeline of 22 companies working in molecular pharming, covering biotherapeutics and vaccine. Since there was no suitable database available in BM for diagnostics, we searched scientific databases (Embase ${ }^{\circledast}$, Scopus ${ }^{\circledast}$, Web of Science ${ }^{\circledast}$ and Pubmed $^{\circledast}$ ) and a patent database (Derwent Innovations Index ${ }^{\circledR}$ ). To clean, classify and analyze the results, we used data mining, descriptive statistics in Microsoft Excel ${ }^{\oplus}$, Tableau ${ }^{\circledast}$ and Vantage Point ${ }^{\circledast}$ software.

Results: We were able to identify the major requirements and methods that could be currently applied in BM for early search and selection of targets. Regarding the TF results, the Medtrack ${ }^{\otimes}$ search retrieved 84 plant-based biotherapeutics (market phase, $\mathrm{N}=1$; phase III, $\mathrm{N}=2$ ) by 14 companies. Transient Expression Systems (TES) were the source of $48.8 \%$ of these products. For plant-based vaccines, five companies had 22 products under research (phase III, $N=1$ ), all of which obtained by TES. For diagnostics, three out of 18 publications mentioned products obtained by TES. Regarding patents, nine out of 1,095 original documents were selected for further analyses due to their relevance. Four of these patents were related to at least one of the 18 original publications, but no products were identified on the specific market.

Conclusion: In general, we demonstrated that TES is a broad range expression system and can be the best choice for many targets. However, the market database and the search of scientific and patents databases did not add to the existing knowledge of vaccines and diagnostics products in market players, respectively. For vaccines and biotherapeutics, the use of scientific and patent information is also necessary to complement the analysis and for diagnostics, other foresight techniques are required. Besides an overall view of trends and potential areas of interest, this work also demonstrates the importance of institutionalizing TF. Further work is necessary to improve the data gathering as well as data analytics.

Keywords: Technology foresight; Plant-based technologies; Biopharmaceutical development 